\title{
The Gaps between Knowing and Doing in Hearing Aid Fitting Management
}

\author{
Soo Hee $\mathrm{Oh}^{1}$, Kyoungwon Lee ${ }^{1,2}$, and Junghak Lee ${ }^{1,2}$ \\ ${ }^{1}$ Audiology Institute, Hallym University of Graduate Studies, Seoul, \\ ${ }^{2}$ Department of Audiology, Hallym University of Graduate Studies, Seoul, Korea
}

Received December 30, 2016

Revised February 14, 2017

Accepted March 26, 2017

Address for correspondence

Junghak Lee, FAAA, BCA, PhD

Department of Audiology,

Hallym University of Graduate Studies,

427 Yeoksam-ro, Gangnam-gu,

Seoul 06197, Korea

Tel $+82-2-2051-4950$

Fax $+82-2-3453-6618$

E-mail1eejh@hallym.ac.kr
Evidence based practice has become an important aspect in many healthcare provisions to improve patient outcomes. Investigating the gaps between knowing and doing in audiology practice will provide future directions for successful service delivery. The purpose of this study was to explore "know-do" gaps related to professional awareness and attitude in hearing aid fitting management (HAFM) by analyzing data of a questionnaire. It consisted of 22 questions focusing on 11 HAFM components. Each component had two sub-questions involving levels of the importance and practice for HAFM. Respondents answered each question based on subjective judgments for their own hearing aid fitting services with a Visual Analogue Scale. A total of 51 responses from hearing care professionals were collected and the scores for knowing and doing were compared. The results showed overall high scores in doing and knowing responses; however, there were significant "know-do" gaps demonstrating higher knowing scores than doing scores across all HAFM components. The largest "know-do" gaps were observed in three components mostly implemented during follow-up sessions which indicate the need to improve their corresponding services and practical guidelines emphasizing the follow-up services.

J Audiol Otol 2017;21(2):120-123

KEY WORDS: Hearing aid fitting management · Professional awareness · Practical guideline.

\section{Introduction}

With growing emphasis, evidence-based practice is widely adopted in many health care provisions including audiology to deliver optimal services [1-4]. It is important for health care service providers to effectively transfer knowledge into practice $[2,5]$ because gaps between knowledge and actual practice may not cause only poor outcomes, but also client dissatisfaction. In general, "knowing" indicates what healthcare professionals know through multiple sources such as research recommendations, scientific evidence, and clinical guidelines; whereas "doing" refers to what practitioners do routinely [2]. Few studies have recognized issues of the gaps between knowledge of health care professionals and their actual practices $[2,5]$ and the "know-do" gaps appear to be issues of "practice/

This is an Open Access article distributed under the terms of the Creative Commons Attribution Non-Commercial License (http://creativecommons.org/licenses/by-nc/4.0/) which permits unrestricted non-commercial use, distribution, and reproduction in any medium, provided the original work is properly cited. service" [6]. Liang [5] addressed factors influencing the disconnections between knowing and doing: scientific and technical complications, increase of chronic conditions, limitations in the healthcare delivery system, and shortages of information technology. In another study, Cochrane, et al. [2] described multiple barriers that impede the "knowledge to action" process: cognitive/behavioral barriers including a lack of knowledge and awareness, attitudinal/rational-emotive barriers, and health care professional/physician barriers. Both studies emphasized that the discrepancies between knowing and doing lead to diminished healthcare outcomes as well as optimal healthcare delivery.

In recent years, hearing aid (HA) markets have increased and best practices provided by hearing care professionals have been strongly related to reductions of hearing handicaps and patient improvements [7]. Among others, there are various worldwide guidelines of HA fitting management (HAFM) to promote best practice with professional knowledge [6]. However, it is uncertain whether hearing care professionals follow 
them or not. In this context, it is meaningful to investigate the "know-do" gaps of HA professionals and to understand the practical HAFM services that the practitioners are providing.

The purpose of this study is to examine "know-do" gaps related to professional awareness and attitude in HAFM [8]. The results will expand our current understanding of the differences between knowledge and practice in HAFM. They will also provide basic information to systematically develop a "know-do" questionnaire as well as relevant standards for better service quality and outcomes of HA provisions.

\section{Subjects and Methods}

\section{Subjects}

The questionnaire was distributed among the attendants of a HA seminar held in Seoul, Korea. Participation was voluntary and no financial compensation for the subjects was provided. A total of 68 anonymous responses was collected without containing the respondents' personal information. All participants were engaged in HA provisions or relevant fields. Questionnaires by students and those with invalid or incomplete responses for more than two items were excluded. The scores of 51 total responses were analyzed by comparing knowing and doing across 11 categories. The Fig. 1 shows all the respondents' occupational information. Thirty six respondents worked in HA centers. The remaining subjects were engaged in hospitals, HA manufactures, and education or research institutes. Three subjects did not provide occupational information.

\section{Questionnaire}

The purpose of the questionnaire was to identify the differ-

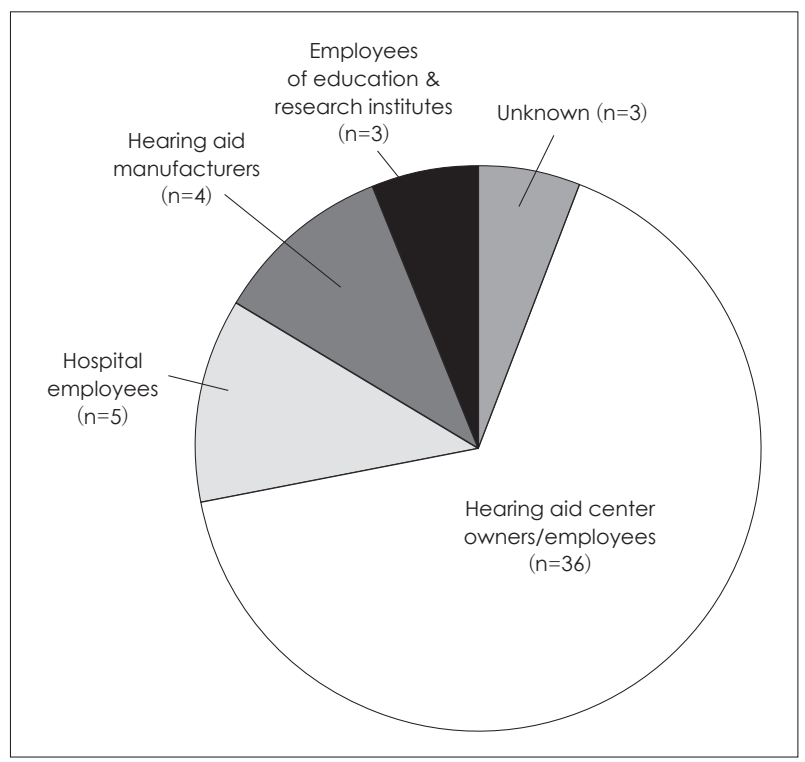

Fig. 1. Occupations of 51 respondents. ences between the respondents' awareness and their actual practices for the HAFM components. In order to directly compare the scores between knowing and doing, a Visual Analogue Scale (VAS) [9] presented as a horizontal line with a continuous value from 0 to 10 was used. The VAS can evaluate the ranges and gaps of subjective judgments. In the questionnaire, a "O" score means that respondents do not realize the importance of a component or do not provide any relevant services at all. A " 10 " score indicates that respondents are fully aware of a component's importance or always provide relevant services. The 11 HAFM components [6] of the questionnaire were as follows: HA counseling, hearing evaluation, HA selection, HA trial, ear impression, HA adjustment, verification of HA adjustment, orientation, outcome measure, auditory training, and comprehensive report. Each component had two knowing and doing response categories. The respondents were instructed to mark a number based on their subjective judgments. For instance, if subjects thought that counseling was the most significant process in HAFM, they marked a 10 score for knowing responses. For doing responses, they could mark from "0" (no service provided) to "10" (service always provided) based on their actually provided services to clients.

\section{Statistical analysis}

Shapiro-Wilk's test indicated that the respondents' scores for each question were not normally distributed $(p>0.05)$. Thus, the Wilcoxon Signed-Rank and Spearman's rank correlation tests were performed to compare the scores between knowing and doing questions by using SPSS version 17 (SPSS Inc., Chicago, IL, USA) for the statistical analyses.

\section{Results}

Total mean scores in knowing (9.1) were 1.2 points higher than doing questions (7.9) $(p<0.05$, Wilcoxon Signed-Ranks test). This trend (higher scores in knowing questions than doing questions) was evident across all 11 components ( $p<0.05$, Wilcoxon Signed-Ranks test). The highest mean score was 9.4 for the hearing evaluation and the lowest was 5.8 for the comprehensive report in doing questions. The highest and lowest mean scores for knowing questions were 9.8 and 8.1 for hearing evaluation and comprehensive report respectively. The mean gap between knowing and doing scores was 1.2 points; auditory training and hearing evaluation showed the largest ( 2.8 points) and smallest ( 0.4 points) gaps, respectively. Fig. 2 displays the differences between knowing and doing responses of the respondents for all components. Table 1 exhibits the mean values of "know-do" gaps across the 11 HAFM components.

To investigate correlations between knowing and doing re- 


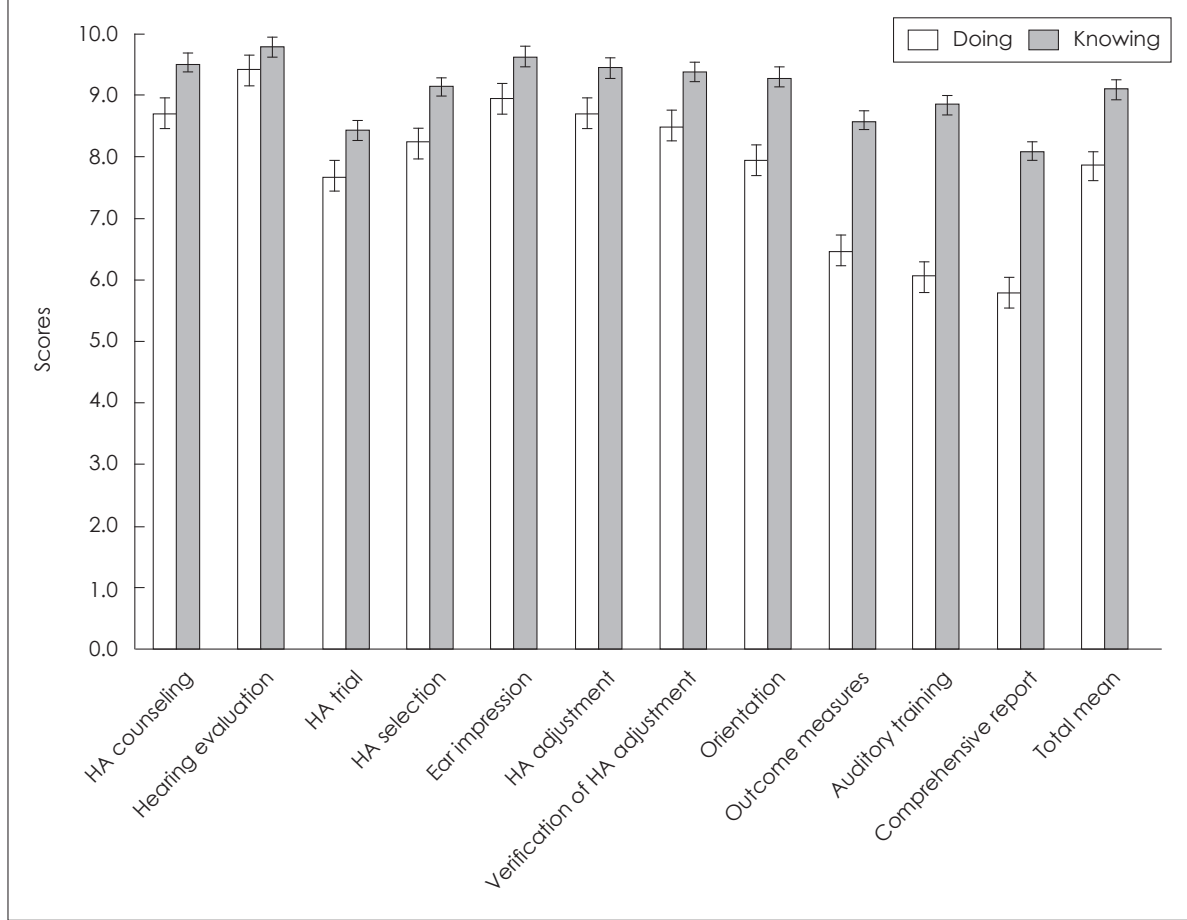

Fig. 2. Differences between knowing and doing responses obtained by 51 respondents across 11 HAFM components. Error bars indicate standard errors. HAFM: hearing aid fitting management, $\mathrm{HA}$ : hearing aid. sponses, Spearman's rank correlations were performed. The results showed positive correlations between knowing and doing total mean scores $(p<0.01$, correlation coefficients: 0.358 ). All components except auditory training ( $p=0.24$, correlation coefficients: 0.238 ) showed positive correlations ( $p<$ 0.05 ) with correlation coefficients 0.4 for comprehensive report and 0.7 for hearing evaluation.

\section{Discussion}

The present study investigated the differences between knowing and doing in HAFM. Fifty-one out of 68 questionnaires were analyzed to compare knowing and doing scores. The results revealed overall high scores for both of them. However, there were significant "know-do" gaps: scores were higher in knowing than in doing for all HAFM components.

Interestingly, the largest "know-do" gaps were observed in three components involving outcome measures, auditory training, and comprehensive report-these are mostly implemented during follow-up sessions [6]. Hearing evaluation exhibited the smallest gaps among all components. In overall, the results indicate that HA professionals recognize the importance of each service well (9.1 mean score), but, they provide fewer practical services (7.9 mean score). The services related to hearing evaluation were better than follow-up services.

There were two main limitations in this preliminary study. First, the questionnaire's construction and validation were not evaluated. Second, the research design was fairly restricted: among others, the sample size and selection criteria were not systematically considered. Nonetheless, the results provided initial insights into improving follow-up services in HAFM and into developing a questionnaire or standardized tool to evaluate the "know-do" gaps of HAFM services.

Significant correlations between knowing and doing scores at moderate levels indicate that providing education, relevant materials and resources, emphasizing the best practice decrease the "know-do" gaps in follow-up services. Improvement of professional awareness and attitude for lifetime services has to be first. For instance, according to the patients' journey model for adults with hearing impairment [10], HA professionals should provide lifetime support for patients. To reduce discrepancies between knowing and doing scores, increasing professional awareness for all HAFM services is necessary through ongoing interactions with clients and by providing practical services on a continuous base. Additionally, active client supports focusing on follow-up services should be emphasized in education programs for HA professionals. As is well known, the high prices of HAs mostly consist of the devices' costs themselves as well as other service fees, including hearing assessment, counseling, fitting, and follow-up [11]. However, HA users often do not fully use the services that they have already paid for [12]. Active supports by HA professionals for the hearing impaired, not only with regard to hearing assessment but also follow-up are expected to reduce the "know-do" gaps, leading client satisfactions.

As previously mentioned, Cochrane, et al. [2] identified the 


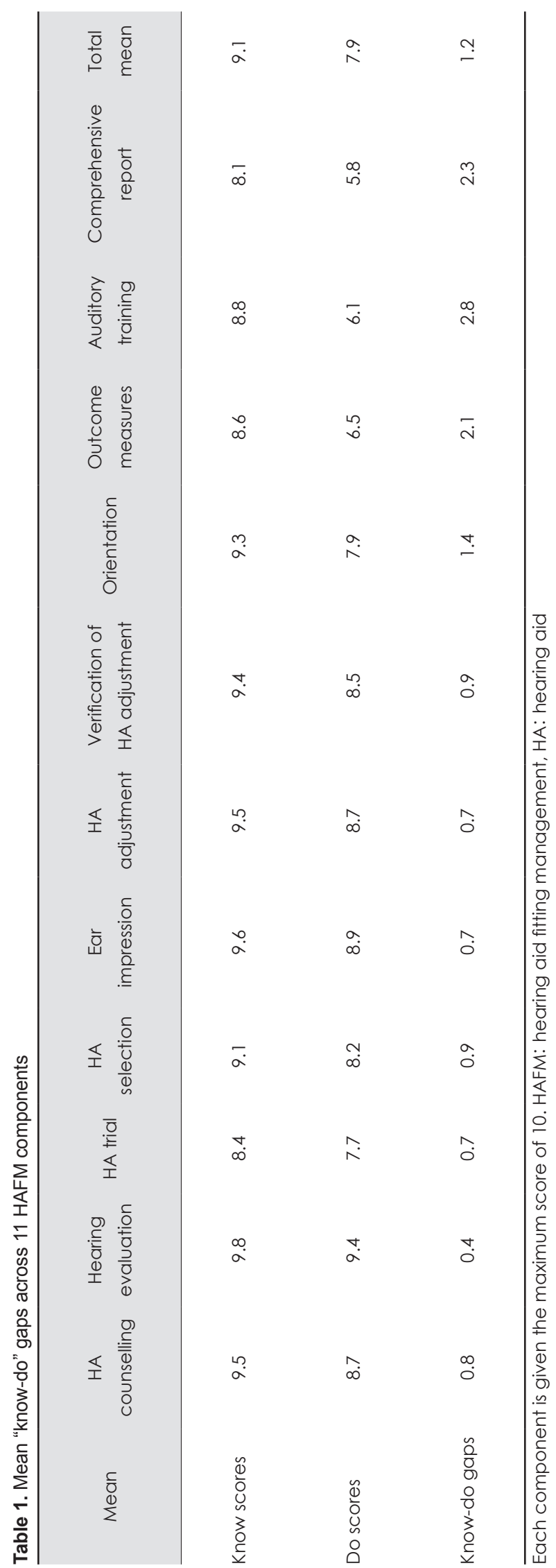

barriers of healthcare providers to lead the gaps between evidence and implementation: cognitive/behavioral, attitudinal/ rational-emotive, healthcare professional/physician, clinical practice guideline/evidence, patient, support/resource, and system/process. In order to decrease these barriers, they introduced a framework explaining ongoing interactions among professionals' knowledge, attitude, and behavior by patient factors, guidelines, and environment. Knowledge and attitude of HA service providers will directly affect their behavioral practices. Thus, continuing education, internationally consented guidelines, and management confirmation will contribute to diminish the knowing-doing gaps in HA services. Future studies focusing on various barriers related to these "know-do" gaps are needed to improve transfer of knowledge to practice, facilitating professional practices in HA provisions.

\section{Acknowledgments}

This work was supported by a grant from the Korean Ministry of Trade, Industry and Energy, Project No. 10049456.

\section{Conflicts of interest}

The authors have no financial conflicts of interest.

\section{REFERENCES}

1) Cox RM. Evidence-based practice in provision of amplification. $J$ Am Acad Audiol 2005;16:419-38.

2) Cochrane LJ, Olson CA, Murray S, Dupuis M, Tooman T, Hayes S. Gaps between knowing and doing: understanding and assessing the barriers to optimal health care. J Contin Educ Health Prof 2007;27: 94-102.

3) Dopson S, Bennett C, Fitzgerald L, Ferlie E, Fischer M, Ledger J, et al. Health care managers' access and use of management research [cited 2017 June 19]. Available from: http://www.netscc.ac.uk/hsdr/ files/project/SDO_ES_08-1808-242_V01.pdf.

4) Moodie ST, Kothari A, Bagatto MP, Seewald R, Miller LT, Scollie $\mathrm{SD}$. Knowledge translation in audiology: promoting the clinical application of best evidence. Trends Amplif 2011;15:5-22.

5) Liang L. The gap between evidence and practice. Health Aff (Millwood) 2007;26:w119-21.

6) Rycroft-Malone J. From knowing to doing-from the academy to practice comment on "the many meanings of evidence: implications for the translational science agenda in healthcare". Int J Health Policy Manag 2014;2:45-6.

7) Kochkin S. MarkeTrak VIII patients report improved quality of life with hearing aid usage [cited 2017 June 19]. Available from: http:// www.betterhearing.org/sites/default/files/hearingpedia-resources/ MarkeTrak\%20VIII\%20Patients\%20report\%20improved\%20quality $\% 20$ of $\% 201$ ife $\% 20$ with\%20hearing\%20aid\%20usage.pdf.

8) $\mathrm{Oh} \mathrm{SH}$, Lee J. General framework of hearing aid fitting management. J Audiol Otol 2016;20:1-7.

9) Crichton N. Information point: Visual Analogue Scale (VAS). J Clin Nurs 2001;10:697-706.

10) Manchaiah VK, Stephens D, Meredith R. The patient journey of adults with hearing impairment: the patients' views. Clin Otolaryngol 2011;36:227-34.

11) Dybala P. Hearing aid prices [cited 2017 June 19]. Available from: http://www.healthyhearing.com/help/hearing-aids/prices.

12) Blustein J, Weinstein BE. Opening the market for lower cost hearing aids: regulatory change can improve the health of older Americans. Am J Public Health 2016;106:1032-5. 